\title{
¿ES POSIBLE CRIAR TIBURONES? EL CASO DE LA PINTARROJA COMUN, SCHROEDERICHTHYS CHILENSIS (CHONDRICHTHYES, SCYLIORHINIDAE)
}

\author{
IS IT POSSIBLE TO BREED SHARKS IN CAPTIVITY? THE CASE OF THE \\ DOGFISH, SCHROEDERICHTHYS CHILENSIS (CHONDRICHTHYES, \\ SCYLIORHINIDAE)
}

\author{
Nicole Piaget ${ }^{1}$, Sebastián Hernández ${ }^{1}$, Julio Lamilla² $\&$ Alonso Vega ${ }^{1,3}$ \\ ${ }^{1}$ Facultad de Ciencias del Mar, Universidad Católica del Norte. Casilla 117, Coquimbo, Chile. \\ Email: nicolepiaget@yahoo.com \\ ${ }^{2}$ Facultad de Ciencias. Universidad Austral de Chile. Casilla 567, Valdivia, Chile. \\ ${ }^{3}$ Centro de Estudios Avanzados de Zonas Aridas (CEAZA)
}

\begin{abstract}
RESUMEN
La crianza de animales es un fenómeno asociado a los orígenes del hombre moderno. Sin embargo, la crianza de especies marinas está relacionada con la producción intensiva en cultivo con fines comerciales. Este estudio tiene como objetivo determinar la factibilidad de crianza de Schroederichthys chilensis, la pintarroja común, a través de experiencias preliminares en ambiente controlado. Esta especie, endémica del Pacífico Sur-oriental, habita desde Perú (Ancón) hasta Chile Austral, preferentemente en aguas someras. Se colectaron cápsulas con huevos de pintarroja del medio natural, los cuales fueron mantenidos en un laboratorio húmedo. Después de la eclosión, las pintarrojas fueron criadas en estanques de 200, 500 y $1000 \mathrm{~L}$ de agua de mar, con flujo de agua constante y temperatura ambiente. Las dimensiones de los estanques utilizados dependieron del número y de la talla de los individuos. Durante el período de crianza en cautiverio las pintarrojas fueron alimentadas con una dieta húmeda, basada en una mezcla de harina de pescado, camarones, jibia y carne de pescado fresco, enriquecida con vitaminas y minerales. Nuestros resultados preliminares sugieren que las pintarrojas pueden ser criadas en cautiverio. La crianza de $S$. chilensis en cautiverio es un precedente para el cultivo de tiburones, los cuales pueden ser dirigidos, entre otras áreas y objetivos, a la investigación, a la conservación de poblaciones nativas y a la educación ambiental.
\end{abstract}

Palabras Claves: Schroederichthys chilensis, tiburones, cultivo, dieta, crecimiento, pintarroja, Chile.

\section{ABSTRACT}

Breeding animals is a phenomenon associated to the origins of the modern man. Nevertheless, breeding marine species is related to the productive scope through intensive cultures in captivity to mainly commercial aims. This study aims to determine the feasibility of culture of the red spotted catshark, Schroederichthys chilensis, through preliminary controlled environment experiences. This species is endemic of the South-Eastern Pacific and lives from Peru (Ancon) to Southern Chile. The egg capsules of red spotted catshark were collected from its natural environment and then were maintained in the wet laboratory. After the hatching of the embryos, the red spotted catshark were maintained in tanks of 200,500 , and $1000 \mathrm{~L}$ of seawater with constant water flow and room temperature. The dimensions of the tanks depended on the number and size of the individuals. During the period of captivity, the red spotted catshark were fed with a moisten diet cradle in a mixture of shrimp, squid and meat of fresh fish, enriched with vitamins and minerals. Our preliminary results suggest that the red spotted catshark can be able to breed in captivity. The breeding of S. chilensis in captivity is a precedent for the culture of sharks, which can be directed to research, conservation of native populations, and environmental education, among other areas.

KEYwords: Schroederichthys chilensis, sharks, culture, diets, growth, redspotted catshark, Chile. 
Es posible criar tiburones?: PIAgEt, N. ETAL

\section{INTRODUCCION}

La crianza de peces en cautiverio está dirigida a incrementar la producción de biomasa a través de cultivos intensivos de especies que presentan un alto riesgo de extinción por sobreexplotación, principalmente aquellas que tienen una alta demanda en los mercados internacionales (Welcomme \& Barg 1999). Durante años, los peces cartilaginosos (Chondrichthyes) han tenido una alta demanda extractiva, con una explotación que en la actualidad se ha intensificado debido a la creciente industrialización de la pesca mundial (FAO 2001). Sin embargo, sólo recientemente se han desarrollado proposiciones de ordenamiento para la conservación y manejo sustentable de las pesquerías de tiburones (Musick 1999, FAO 2001). Los peces cartilaginosos son muy sensibles a la sobreexplotación debido a su particular biología, caracterizada principalmente por un lento crecimiento, edad tardía de la primera madurez sexual, baja fecundidad y largo período de gestación (Camhi et al. 1998). Este ciclo de vida, típico de depredadores de alto nivel trófico de larga vida, aumenta la vulnerabilidad a la sobreexplotación pesquera poniéndolos en riesgo de extinción (Musick 2004). El Plan de Acción Internacional para Tiburones de la FAO reconoce esta vulnerabilidad a la pesca identificando varias amenazas principales tales como: (a) la sobreexplotación pesquera, (b) la pesca accidental (Bycatch), (c) la contaminación y (d) la presión demográfica humana en el litoral (FAO 2001). Es en este contexto, que este trabajo propone la crianza en cautiverio de tiburones para investigación biológica y conservación usando como modelo una especie litoral de Chondrichthyes muy común del Pacífico sudamericano, pero que indudablemente puede ser afectada por algunas de estas amenazas.

La pintarroja común Schroederichthys chilensis (Guichenot, 1848) es un elasmobranquio endémico del Pacífico Sur-oriental, con una distribución latitudinal desde Perú (Ancón) hasta Chile Austral habitando aguas someras del sublitoral rocoso hasta los $50 \mathrm{~m}$ de profundidad (Compagno 1984, Chirichigno \& Cornejo 2001). En ambientes temperados, esta especie es un habitante frecuente de las praderas de algas laminariales (Macrocystis spp y Lessonia trabeculata), donde encuentra refugio y alimento (Vásquez 1989). De acuerdo a Fariña \& Ojeda (1993), S. chilensis es un importante depredador especialista del submareal rocoso con una dieta muy conservadora compuesta en su mayor parte por crustáceos decápodos, principalmente el camarón de roca Rhynchocinetes typus. Aunque en el sur de Chile se ha descrito que también consume sipunculidos (Bahamonde 1952). La pintarroja común utiliza como estrategia reproductiva la oviparía (Leible et al. 1981) y después de la fecundación deposita los huevos en cápsulas con zarcillos que se enrollan a las frondas y los estipes de las algas laminariales (Vásquez 1989) durante las agregaciones reproductivas (Miranda 1980). En Chile, existen reportes de captura accidental (Bycatch) de la pintarroja común durante las faenas de pesca con arrastre y espinel de distintas especies pelágicas y de fondo (Pérez et al. 2004), también ha sido considerada dentro de las pesquerías para la producción de harina o utilizada como carnada para la pesca de centolla y centollón (Illanes 2004). Por otra parte, el incremento exponencial y la expansión regional de la actividad extractiva de laminariales submareales en el centronorte de Chile (Vásquez 1999) sugieren un riesgo potencial para S. chilensis en el futuro cercano. La extracción de plantas completas y la explotación de las praderas de estas algas han disminuido significativamente el hábitat preferentemente utilizado por la pintarroja para las actividades alimenticias y de reproducción. Así, el desconocimiento de distintos aspectos biológicos (por ejemplo: crecimiento de juveniles y adultos, y edad de primera madurez sexual), ecológicos (por ejemplo: distribución espacio-temporal de juveniles) y conductuales de $S$. chilensis, difíciles de observar in situ pero importantes para entender los mecanismos de persistencia poblacional de este depredador del sistema submareal temperado de Sudamérica (Miranda 1980, Vásquez 1989, Fariña \& Ojeda 1993), sumado al riesgo potencial causado por la fragmentación de su hábitat, son también justificaciones válidas para realizar la crianza de esta especie en cautiverio.

Un requisito importante para criar peces en cautividad está relacionado con la alimentación, proporcionándoles dietas adecuadas para su óptimo desarrollo (Welcomme \& Barg 1999). Los hábitos alimenticios de $S$. chilensis son específicos, consumiendo casi exclusivamente camarones de roca (Fariña \& Ojeda 1993). Sin embargo, es posible encontrar símiles alimenticios para la crianza de $S$. chilensis en cautiverio que sean de bajo costo y de fácil adquisición en el mercado, y que eviten el uso de especies incluidas en su espectro trófico natural. 
Gayana 69(1), 2005

\section{MATERIALES Y METODOS}

Colección DE HUEVOS Y CONDICIONES DE CRIANZA DE LAS PINTARROJAS EN CAUTIVERIO

En la localidad de Chungungo (latitud $29^{\circ} 37^{\prime} \mathrm{S}$, longitud $71^{\circ} 19^{\prime} \mathrm{W}$ ) durante julio del 2002, se recolectaron mediante buceo apnea 186 cápsulas con huevos de $S$. chilensis adheridas a estipes y frondas de Lessonia trabeculata ubicadas a $10 \mathrm{~m} \mathrm{de}$ profundidad. Las cápsulas con los huevos fueron mantenidas hasta la eclosión, en un sistema de circulación abierta instalado en el laboratorio húmedo de la Universidad Católica del Norte, usando acuarios de $10 \mathrm{~L}$ con agua de mar microfiltrada $(50 \mu)$ a temperatura ambiente.

La eclosión de los huevos ocurrió después de siete meses, obteniéndose un total de 60 pintarrojas juveniles que fueron mantenidas en un estanque de 200 L. Posteriormente, de acuerdo al incremento en tamaño y a la mortalidad de algunos individuos, las pintarrojas fueron trasladadas a un estanque de 500 L (35 individuos). Durante el último período de crianza las siete pintarrojas supervivientes fueron mantenidas en un estanque de $1000 \mathrm{~L}$. Todos los estaques utilizados para la crianza de las pintarrojas fueron rectangulares y blancos, con sistemas de circulación abierta manteniendo un flujo constante de agua de mar $\left(2 \mathrm{~L} \mathrm{~min}^{-1}\right)$. En los estanques, la temperatura mantuvo una estacionalidad anual con máximos en verano $\left(19-20^{\circ} \mathrm{C}\right)$ y mínimos en invierno $\left(12-13^{\circ} \mathrm{C}\right)$ durante los casi tres años de crianza (2003-2005). La salinidad del agua de mar fue cercana a $\operatorname{los} 35,0 \pm 0,5 \%$. Finalmente, los estanques fueron cubiertos con malla negra para asemejar la penumbra del ambiente submareal dominado por huirales, para minimizar así el estrés de las pintarrojas causado por el cautiverio.

Dietas UTILIZADas PARA LA ALIMENTACIÓN DE LAS PINTARROJASEN CAUTIVERIO

Durante el período de crianza, las pintarrojas fueron alimentadas con tres diferentes dietas húmedas con el mismo porcentaje de contenido proteico (69\%), basadas en una mezcla de carne fresca de pescado, jibia y camarón, que varían en la presencia y/o proporciones (Tabla I). A esta base de fuente proteica se le agregó harina y aceite de pescado, vitaminas y minerales (premix) e inmunoestimulantes en cantidades similares (Tabla I). Antes de preparar las dietas, como una forma de prevenir distintas infecciones, se congeló la carne fresca por un día. La preparación de las dietas se realizó primero juntando los ingredientes secos (harina, premix e inmunoestimulantes), posteriormente se agregó aceite de pescado y, finalmente, la carne fresca molida conformando un sólido homogéneo. Luego de elaboradas, las dietas se conservaron congeladas en forma de bolas de 200 g aproximadamente. Las raciones alimenticias entregadas a las pintarrojas fueron desmenuzadas en porciones proporcionales al tamaño de la boca de los individuos. El alimento fue entregado cada dos días ad livitum, para evitar conductas agresivas. Ninguna de estas dietas fue evaluada experimentalmente durante la crianza de las pintarrojas en cautiverio. En cambio, se realizó una evaluación cualitativa de la aceptación de las distintas dietas por parte de las pintarrojas.

TABLA I. Composición de las tres dietas húmedas utilizadas para la alimentación de Schroederichthys chilensis criados en cautiverio.

TABLE I. Composition of the three diets utilized in the feeding of Schroederichthys chilensis breeds in captivity.

\begin{tabular}{lccc}
\hline \multicolumn{1}{c}{ Fuente Energética } & Dieta 1 & Dieta 2 & Dieta 3 \\
\hline Jibia (Doscidiscus gigas) & $50 \%$ & $40 \%$ & - \\
Jurel (Trachurus murphyi) & - & $29 \%$ & $69 \%$ \\
Camarón (Heterocarpus reedi) & $19 \%$ & - & - \\
Harina de pescado (San Jose S.A.) & $27 \%$ & $27 \%$ & $27 \%$ \\
Aceite de pescado (San Jose S.A.) & $2 \%$ & $2 \%$ & $2 \%$ \\
Premix vitaminico (Veterquímica S.A.) & $1 \%$ & $1 \%$ & $1 \%$ \\
Inmunoestimulantes ( $\beta$-glucanos; Despro S.A.) & $1 \%$ & $1 \%$ & $1 \%$ \\
\hline
\end{tabular}


RELACIÓN LONGITUD-PESO, CRECIMIENTO Y MORTALIDAD DE LAS PINTARROJASEN CAUTIVERIO

Durante el período de estudio se realizaron cinco muestreos de las pintarrojas para estimar (a) la relación longitud-peso, (b) la mortalidad y (c) la tasa de crecimiento de hembras, machos y ambos sexos juntos. En cada muestreo la relación longitud-peso fue estimada midiendo la longitud total de los individuos desde el inicio de la boca hasta el final de la aleta caudal, y posteriormente cada uno fue pesado en una balanza semi-analítica Gram Precision GX1500 (precisión $\pm 0.1 \mathrm{~g}$ ).

La tasa de crecimiento porcentual acumulada para cada uno de los muestreos fue evaluada usando la metodología de Ricker (1979) en función de la longitud total de 4 hembras y 5 machos marcados con marcas de la línea Dennison ${ }^{\circledR}$. Durante el último muestreo tres machos murieron, mientras que todas las hembras sobrevivieron.

El porcentaje de mortalidad fue estimado para cada muestreo. La mortalidad de pintarrojas fue separada en dos tipos principales: (a) Deficiencias del cultivo, este tipo de mortalidad ocurrió durante cortes involuntarios del flujo de agua corriente en el laboratorio. Esta interrupción produjo un incremento significativo en la temperatura del agua de mar en los estanques disminuyendo la capacidad respiratoria de las pintarrojas, que junto a los desechos fecales y excreción de los propios individuos produjeron la muerte de algunos individuos. (b) Comportamiento, este tipo de mortalidad fue dividida en comportamiento de escape y de ataque. Cuando las pintarrojas saltaban cayendo fuera del estanque y morían durante la noche, fue considerado un comportamiento de escape. En cambio, el comportamiento de ataque ocurrió cuando algunas pintarrojas mordieron las agallas de otras, produciendo heridas que impedían tragar el alimento, que a la larga producen la muerte de los individuos por inanición.

\section{RESULTADOS Y DISCUSION}

Viabilidad DE hUEVOS UTILIZADOS PARA LA CRIANZA DE PINTARROJASEN CAUTIVERIO

El problema inicial de la crianza de las pintarrojas en cautiverio esta relacionado con el período de incubación de los huevos, que fue de aproximadamente siete meses. Este tiempo coincide con lo reportado por Hernández (2003), quien determinó que el desarrollo embrionario de $S$. chilensis fue cercano a los 211 días, pero con un desfase de varios días en la eclosión de los huevos y liberación de los juveniles. El 32.2\% (equivalente a 60 juveniles) de las cápsulas colectadas de un total de 186 terminaron el desarrollo embrionario con la eclosión de los huevos en los acuarios incubadores, con una relación de una cápsula eclosionada por cada tres colectadas. Esta relación en la supervivencia de los embriones de pintarroja puede ser explicada en parte por la actividad de traslado desde el sitio de colecta al laboratorio y por la manipulación de las cápsulas durante el período de desarrollo embrionario (Hernández 2003). Se desconoce la viabilidad natural de los huevos de $S$. chilensis, aunque un importante factor de mortalidad de cápsulas es causado por el desprendimiento de plantas completas de Lessonia trabeculata que varan en la costa (Vásquez 1989).

DieTaS HÚMEdas UTILIZADAS PARA LA ALIMENTACIÓN DE LASPINTARROJAS EN CAUTIVERIO

En el ambiente natural, las pintarrojas consumen preferentemente crustáceos decápodos (Fariña \& Ojeda 1993). En Coliumo, varios especímenes adultos de S. chilensis fueron capturados para efectuar estudios hematológicos. Durante el período de aclimatación, los individuos fueron alimentados con trozos de pescados y/o moluscos frescos, o con pellet para salmonídeos (Valenzuela et al. 2003). En nuestro estudio, las pintarrojas en cautiverio aceptan, consumen y digieren las tres dietas preparadas para su alimentación. La diferencia más importante entre las tres dietas preparadas fue la incorporación de camarón en una de éstas (Dieta 1; Tabla I). Esta dieta fue preparada para alimentar a los juveniles de pintarroja durante los primeros seis meses después de la eclosión de los huevos, la cual fue rápidamente aceptada y consumida, pero tiene el mayor costo económico. La dieta elaborada con una base proteica de jibia y jurel (Dieta 2; Tabla I) es mucho más económica, y fue ampliamente aceptada por los individuos de un año de edad, sin necesidad de un periodo de aclimatación previo entre una y otra dieta. Durante los últimos meses del estudio, las pintarrojas aceptaron y consumieron la dieta preparada a base de carne fresca de jurel (Dieta 3), la más económica de todas (Tabla I). A pesar de la amplia aceptación de las pintarrojas a las dietas húmedas en base a camarón/ jibia/ jurel, es necesario 
realizar estudios para determinar si una o todas estas dietas cumplen eficientemente con los requerimientos nutricionales y energéticos específicos de esta especie. Esto es un antecedente básico para la crianza de peces teleósteos en cautiverio (Welcomme \& Barg 1999). Otra opción particular paraS. chilensis, sobre todo si se desea realizar repoblamiento, es ofrecer presas vivas de crustáceos decápodos, o por razones de palatabilidad preparar una dieta húmeda usando como alimento base al camarón de roca $R$. typus (Fariña \& Ojeda 1993). Sin embargo, los camarones de roca son difíciles de colectar in situ, y tienen un alto precio en el mercado.

RELACIÓN LONGITUD-PESO, CRECIMIENTO Y MORTALIDAD DE LAS PINTARROJASEN CAUTIVERIO

De los 60 juveniles de pintarroja obtenidos de las cápsulas 37 fueron hembras y 23 machos, con una razón $\mathrm{H}: \mathrm{M}$ de 1.6:1. Al comparar hembras y machos, ninguna diferencia en los tamaños fue detectada, y el incremento fue similar para ambos sexos durante todo el período de crianza de las pintarrojas en cautiverio (Tabla II). Durante el último muestreo, una de las hembras presentó marcas en la cloaca causadas por intentos de copula (observación personal), un indicador preliminar de actividad sexua (Conrath 2004). En ensayos preliminares, otras hembras muertas de pintarroja mantenidas en cautiverio y de tamaños equivalentes han tenido marcas muy similares. Es posible que en cautiverio, la pintarroja común alcance la primera edad de madurez sexual al comienzo del tercer año de vida, particularmente en hembras con tamaños sobre los $40 \mathrm{~cm}$ de longitud y $250 \mathrm{~g}$ de peso, respectivamente. La mayoría de las hembras de S. chilensis con tallas sobre los $40 \mathrm{~cm}$ de longitud, capturadas desde el ambiente natural, presentan en los oviductos 1 a 2 cápsulas con huevos fecundados (Fariña \& Ojeda 1993). Sin embargo, para tiburones la primera edad de madurez sexual no indica que estén aptas para la reproducción (Conrath 2004).

TABLA II., Longitud pomedio de hembras, machos y totales de Schroederichthys chilensis criados en cautiverio por fecha de muestreo. $\mathrm{N}$ indica el $\mathrm{n}^{\circ}$ de individuios

TABLE II. Mean length of female, male and total of Schroederichthys chilensis breeds in captivity per samplin date. N indicates number of individuals.

\begin{tabular}{lcccccc}
\hline $\begin{array}{l}\text { Fecha de } \\
\text { muestreo }\end{array}$ & \multicolumn{2}{c}{ Membras } & \multicolumn{3}{c}{ Machos } & \multicolumn{2}{c}{ Totales } \\
\hline 16-Ene-03 & $7,7(0,2)$ & 37 & $6,9(0,8)$ & 23 & $8,6(0,8)$ & 60 \\
08-Jun-03 & $26,4(1,7)$ & 8 & $25,2(2,3)$ & 6 & $25,7(2,0)$ & 13 \\
09-Ago-03 & $28,4(1,0)$ & 8 & $27,2(0,8)$ & 6 & $27,8(1,1)$ & 13 \\
26-Oct-04 & $36,7(3,8)$ & 8 & $39,9(2,7)$ & 6 & $37,9(3,7)$ & 13 \\
18-Mar-05 & $42,3(3,4)$ & 5 & $41,5(2,1)$ & 2 & $42,1(2,9)$ & 7 \\
\hline
\end{tabular}

Es posible que a pesar de tener una conducta sexual reproductiva, las pintarrojas en cautiverio todavía presenten inmadurez de los órganos reproductores en la mayoría de los individuos. En función de estos datos, esperamos que este año las pintarrojas copulen, para posteriormente obtener crías vivas en cautiverio. Estudios previos han logrado que una hembra de S. chilensis depositara cápsulas fecundadas en cautiverio (Hernández 2003).

Una función potencial describe la relación entre la longitud total y la biomasa de las pintarrojas criadas en cautiverio (Fig. 1). La interacción entre sexo y longitud total y la influencia de estos parámetros sobre el peso del individuo fue comparada usando ANCOVA, sobre los datos transformados al logaritmo natural. Ninguna diferencia fue detectada entre sexos, indicando que para una misma longitud (Tabla II), el peso fue similar para ambos sexos (Fig.1). Resultados similares han sido descritos para otros Scyliorhinidae (Ivory et al. 2004, Musick 2004)

Las tasas de crecimiento de las pintarrojas marcadas no difieren significativamente entre sexos durante los casi tres años de crianza en cautiverio (Fig. 2). 


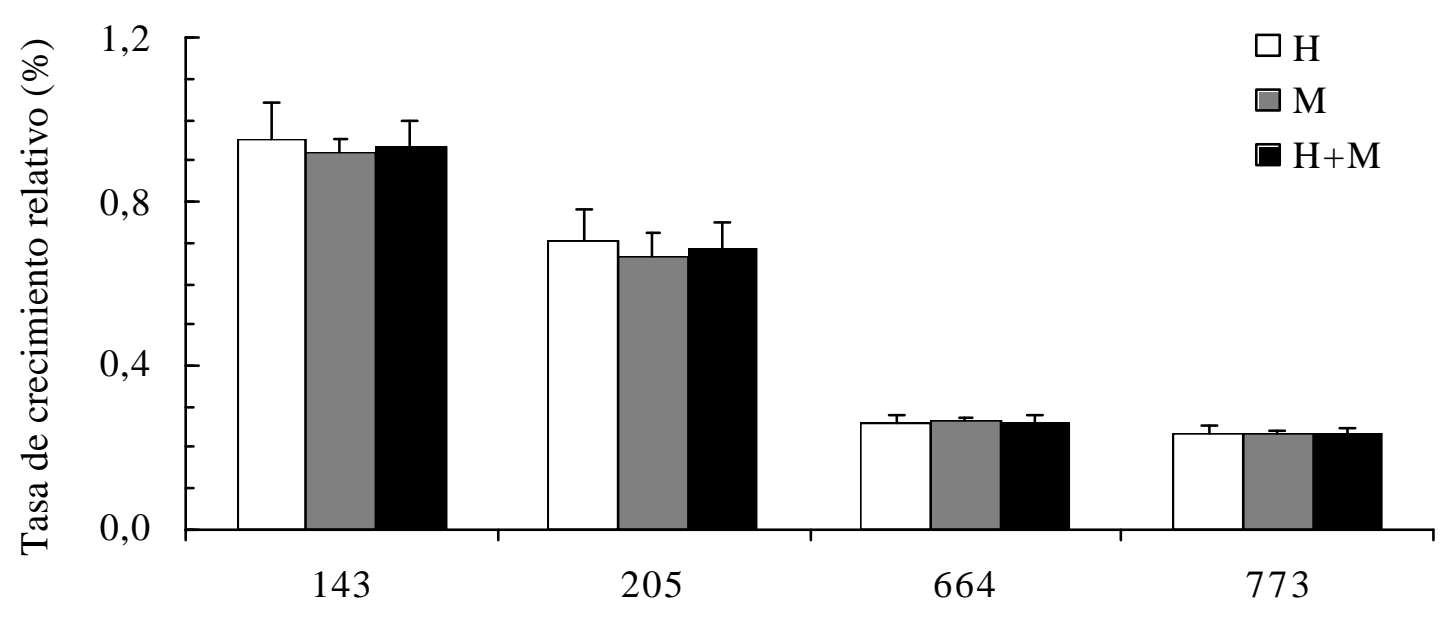

Días desde la eclosión del huevo

Figura 1. Relación longitud-peso de (A) hembras, (B) machos y (C) total de Schroederichthys chilensis criados en cautiverio. En el gráfico se indica la función potencial para cada curva.

FIGURE 1. Length-weight of (A) females, (B) males and (C) total of Schroederichthys chilensis breeds in captivity. In the graphic is indicated power function for each curve.

Aunque las tasas de crecimiento durante el primer año de vida fueron el doble más alto que las obtenidas durante el segundo año (Fig. 2).

La mortalidad durante los primeros meses de crianza de pintarrojas fue alta (Tabla III). El mayor porcentaje de mortalidad fue debido a las deficiencias propias del sistema de circulación de agua en el estanque (Tabla III). Situaciones como la obstrucción de los filtros y tomas de agua, y cortes eléctricos produjeron cortes del flujo de agua que repercutió en la supervivencia de los juveniles de pintarroja. Sin embargo, aspectos conductuales como escape fuera del estanque por saltos en los bordes de la malla protectora y el ataque entre ellos también fueron causas importantes de mortalidad juvenil (Tabla III). Períodos de inanición por más de tres días promovieron conductas agresivas entre los juveniles, con repercusiones negativas en los individuos de menor tamaño. Por esto, fue necesario alimentar a las pintarrojas ad livitum cada dos días. Además, es posible que la estacionalidad de las variables ambientales (por ejemplo: temperatura) afecte también el comportamiento y hábito alimenticio de las pintarrojas, como ha sido sugerido para otros Scyliorhinidae (Laptikhovsky et al. 2001). Por sobre los seis meses de edad, las pintarrojas presentan muy bajas mortalidades (Tabla III). Durante el último muestreo, la mortalidad de cuatro machos fue debido a comportamientos de ataque (Tabla III) por parte de las hembras. Estos individuos presentaron daños en las aletas (dorsales y laterales) y mordeduras en las branquias, produciendo la pérdida de apetito y la posterior muerte por inanición. Se desconoce el comportamiento reproductivo en pintarrojas en el ambiente natural, y los ataques de hembras sobre machos podrían estar asociados a la supuesta actividad reproductiva de las hembras. En tiburones, las hembras usan el comportamiento agresivo como un mecanismo pre-copulador (Conrath 2004).

Nuestros resultados indican que las pintarrojas pueden ser criadas en cautiverio. Sin embargo, mucha más investigación es necesaria todavía. En este contexto se debería estimar la denso-dependencia asociada a la capacidad de carga de los estanques. Otras dietas que apunten a aumentar las tasas de crecimiento relativo, y la observación de los fenómenos conductuales propios del cautiverio son relevantes para la crianza de tiburones. En conclusión, la crianza de esta especie es un precedente para el cultivo de tiburones, la cual puede ser dirigida a la investigación (médica, bioindicadores de contaminación, etc.), a la conservación de poblaciones nativas (sobreexplotación) y a la educación ambiental. 

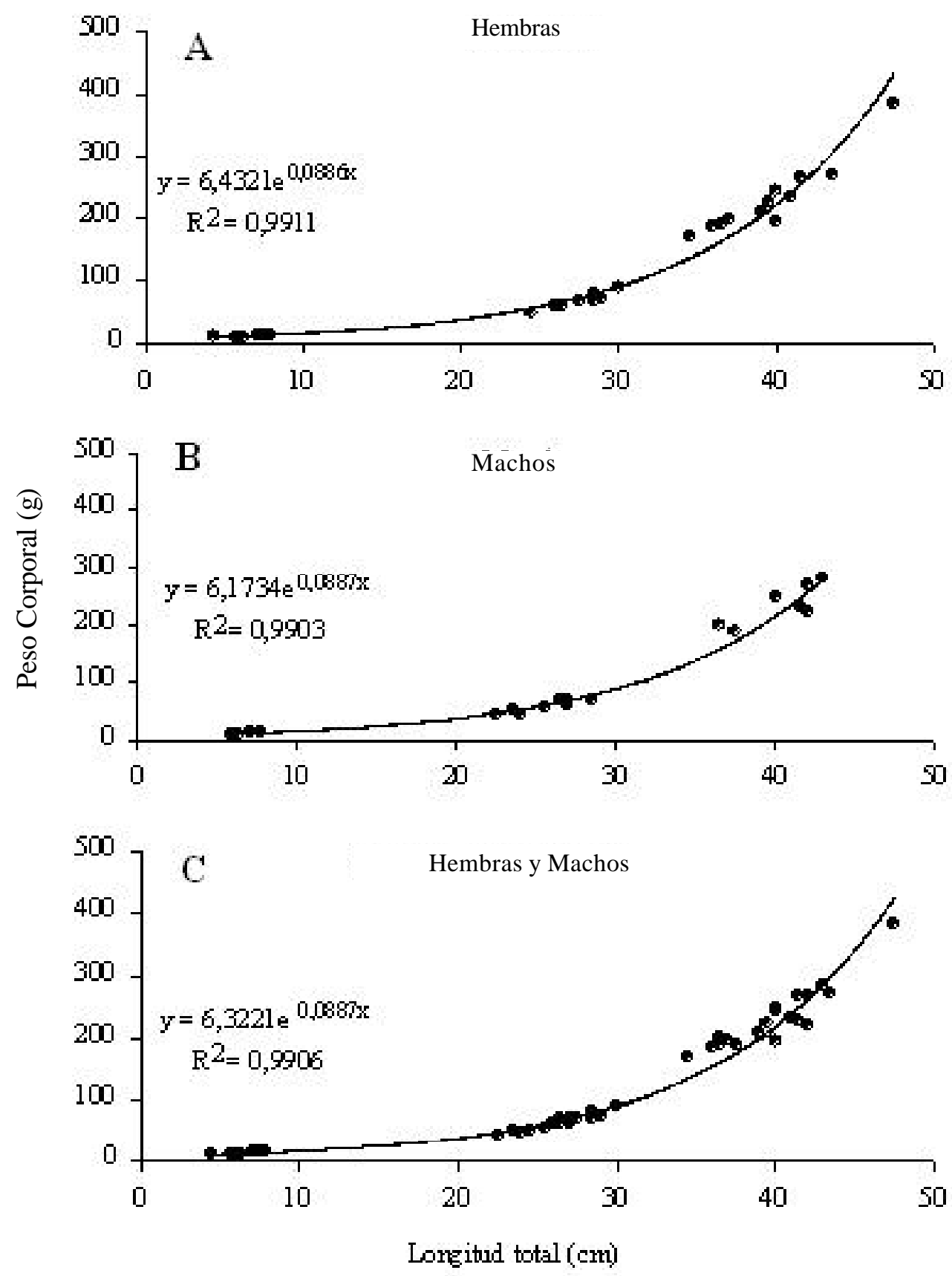

FIGURA 2. Tasa de crecimiento relativo de hembras $(\mathrm{H})$, machos $(\mathrm{M})$ y total $(\mathrm{H}+\mathrm{M})$ de Schroederichthys chilensis criados en cautiverio.

FIGURE 2. Relative growth rate of females (H), males (M) and total $(\mathrm{H}+\mathrm{M})$ of Schroederichthys chilensis breeds in captivity. 
Es posible criar tiburones?: PIAgEt, N. ETAL

TABLA III. Mortalidad porcentual de Schroederichthys chilensis criados en cautiverio por fecha de muestreo. N indica número de individuios

TABLE III. Percentage of mortality of Schroederichthys chilensis breeds in captivity per sampling date. $\mathrm{N}$ indications number of individuals

\begin{tabular}{lccccc}
\hline $\begin{array}{l}\text { Fecha de } \\
\text { muestreo }\end{array}$ & N & Mortalidad (\%) & $\begin{array}{c}\text { Deficiencias } \\
\text { del Cultivo }(\%)\end{array}$ & $\begin{array}{c}\text { Comportamiento } \\
\text { de escape }(\%)\end{array}$ & $\begin{array}{c}\text { Comportamiento } \\
\text { de ataque }(\%)\end{array}$ \\
\hline 16-Ene-03 & 60 & 0 & - & - & - \\
08-Jun-03 & 13 & 78 & 49 & 26 & 26 \\
09-Ago-03 & 13 & 78 & - & - & - \\
26-Oct-04 & 13 & 78 & - & - & - \\
18-Mar-05 & 7 & 88 & - & - & 100 \\
\hline
\end{tabular}

\section{AGRADECIMIENTOS}

Se agradece al Laboratorio de Peces de la Universidad Católica del Norte por proporcionar la infraestructura y los ingredientes para la preparación de las dietas de los tiburones, en especial la disposición de McS (c) Marcia Oliva, McS (c) Bartolo Concha y al Centro de Producción de la Facultad de Ciencias del Mar por la disponibilidad de espacio para mantener a los tiburones en cautiverio. Además, se agradecen los comentarios de los dos revisores anónimos.

\section{BIBLIOGRAFIA}

Bahamonde, N. 1957. Alimentación de la Pintarroja (Halaelurus chilensis (Guichenot), 1848). Inv. Zool. Chilenas 1:3-5.

Camhi, M. S., S. Fowler, J. Musick, A. Bräutigan \& S. FordHAM. 1998. Sharks and their relatives ecology and conservation. Occasional Paper of the IUCN Species Survival Commission 20 pp.

Chirichigno, N. \& M. Cornejo. 2001. Catálogo comentado de los peces marinos del Perú. Instituto del Mar del Perú. Callao. 314 pp.

Compagno, L. 1984. FAO Species catalogue. Vol. 4. Sharks of the World. An annoted and illustrated catalogue of sharks species known to date. Part 2. Carcharhiniformes. FAO Fish. Synop. (125) vol 4. pt. 2:251-655.

ConRath, C.L. 2004. Reproductive Biology. In: Musick, J.A. \& R. Bonfil (Eds). Elasmobranch Fisheries Management Techniques. APEC Secretariat Singapore: 133-164.
FAO 2001. Documento técnico $\mathrm{N}^{\circ} 4$. La ordenación pesquera. 1. Conservación y ordenación del tiburón Orientaciones técnicas para la pesca responsable. FAO. http://www.fao.org/documents/ DOCREP/ 005/X8692S/x8692s01.htm.

FARIÑA, J \& P. OJEDA. 1993. Abundance, activity and trophic patterns of the red spotted catshark, Schroederichthys chilensis, on the pacific temperate coast of Chile. Copeia 1992 (2): 545-549.

Hernández, S. H 2003. Descripción del desarrollo embrionario de la pintarroja común, Schroederichthys chilensis (Guichenot 1848) (Chondrichthyes: Scyliorhinidae). Tesis para optar al Título de Biólogo Marino. Departamento de Biología Marina. Facultad de Ciencias del Mar. Universidad Católica del Norte. 39 pp.

ILLANES, D. 2004. Regulación en el abastecimiento de carnada utilizada en la pesquería de la centolla y centollón entre los años 2000 y 2002 en la XII Región. Tesis para optar al Titulo de Biólogo Marino. Departamento de Biología Marina. Facultad de Ciencias del Mar. Universidad Católica del Norte. $45 \mathrm{pp}$.

Ivory, P., F. Jeal \& C. P. Nolan. 2004. Age determination, growth and reproduction in the Lesser-spotted dogfish, Scyliorhinus canicula (L.). e-Journal of Northwest Atlantic Fishery Science 35(2).

Laptikhovsky, V. V., A. I., Arkhipkin \& A. C. Henderson. 2001. Feeding habits and dietary overlap in spiny dogfish Squalus acanthias (Squalidae) and narrowmouth catshark Schroederichthys bivius (Scyliorhinidae). J. Mar. Biol. Ass. U. K. 81: 10151018.

Leible, M. D., D. M. D ittus \& C. G. G. Belmar. 1981. Atlas anatómico de la pintarroja Schroederichthys chilensis (Guichenot 1874) (Chondrichthyes: Scyliorhinidae). Pontificia Universidad Católica de Chile, Departamento de Biología y Tecnología del 
Mar. Vol. 1.

Miranda, O. 1980. Agregación sexual deSchroederichthys chilensis (Guichenot 1848) durante la parición. Noticiario Mensual, Museo Nacional de Historia Natural. Año XXIV N² 286: 7-9.

Musick, J.A. 1999. Ecology and Conservation of LongLived Marine Animals. American Fisheries Society Symposium 23:1-10.

Musick, J. A. 2004. Introduction: Management of sharks and their relatives (Elasmobranchii). In: Musick, J.A. \& R. Bonfil (Eds). Elasmobranch Fisheries Management Techniques. APEC Secretariat Singapore: $1-9$.

Pérez, A., A. Bushman \& C. Cortés. 2004. By catch en Chile: Una aproximación a los efectos de la pesca sobre la diversidad marina. Oceana 4:1-76.

Ricker, N. E. 1979. Growth rates and models. Fish Physiology. VIII Series. Chapter 11. Academia Press:
677-743.

Valenzuela, A., C. Oyarzún \& B. Silva. 2003. Células sanguíneas de Schroederichthys chilensis (Guichenot 1848) (Chondrichthyes: Scyliorhinidae): la serie blanca. Gayana (Concepción) 67(1): 130-137.

VÁsQuEZ, J. A. 1989. Estructura y organización de huirales submareales de Lessonia trabeculata. Tesis doctoral Universidad de Chile, Facultad de Ciencias. $261 \mathrm{pp}$.

VÁsqueZ, J. A. 1999. The effects of harvesting of brown seaweeds: a social, ecological and economical important resource. World Aquaculture 30: 19-22.

Welcomme, R.I. \& U. Barg. 1999. Documento técnico $\mathrm{N}^{\circ} 5$. _Orientaciones Técnicas para la Pesca Responsable Desarrollo de la Acuicultura. FAO. http://www.fao.org/documents/ docrep/003/ w4493s/w4493s00.htm 